\title{
TIME-INDEPENDENT STOCHASTIC DESIGN SENSITIVITY ANALYSIS OF STRUCTURAL SYSTEMS WITH SECOND-ORDER ACCURACY
}

\author{
HANNA WEBER \\ West Pomeranian University of Technology in Szczecin, Faculty of Civil Engineering and Architecture, Poland \\ e-mail: weber@zut.edu.pl
}

\begin{abstract}
In the paper, the static sensitivity of complex structures with respect to random design parameters is presented. Using the adjoint system method, based on the mean-point second-order perturbation method, the first two probabilistic moments of time-independent sensitivity are formulated with means and cross-covariances of random design parameters as the input data. It enables one to obtain the second-order accuracy of the solution. The presented formulations are illustrated by a number of numerical examples. The influence of finite element mesh density for the obtained results is discussed using the analysis of the spatial bar dome.

Keyword: design sensitivity, statics, random parameter, finite element
\end{abstract}

\section{Introduction}

Since the beginning of mankind, humans have had aspirations to overcome their weaknesses and limitations. It is the element of our nature, which became the basis of development in all fields of life. In the civil engineering there are amazing objects that crossed existing barriers of height, span and slenderness. These types of structures can not be computed by analytical methods because of complexity of the system. Hence, the Finite Element Method (FEM) has become the basis of contemporary structure analysis (Bathe, 1982; Zienkiewicz and Taylor, 1991; Kincayd and Cheney, 2002). It is an approximate method which allows one to obtain highly precise results with a properly dense FEM mesh.

A significant element of correct design is to model an object to reflect reality as closely as possible while taking into account many factors including connections, materials, external loads, etc. (see Leet et al., 2010). Engineers' aim is to create programs for optimal design of new or strengthen the existing structures. It involves findings the best solutions bearing in mind many different aspects such as the maximum load, allowable displacement, cost, time and possibility of execution, etc. (Niczyj, 2003; Choi and Kim, 2010). A very important case is to find an answer to the question of how the changing of design parameters affects the structural response. It is a subject of study of sensitivity analysis that can be found in (Haug et al., 1986; Mroz and Haftka, 1986; Hien and Kleiber, 1989; Drewko and Hien, 2005; Ding et al., 2012; Mroz and Bojczuk, 2012; Choi and Kim, 2013). This type of computations by using deterministic variables has been extensively discussed in the literature. However, it has been proved that in systems with many degrees of freedom (MDOF), even small uncertainties in structural parameters or external loads may have a significant impact on the work and load capacity of the system. The stochastic analysis (Adomian, 1983; Hisada and Nakagiri, 1981; Spanos and Ghanem, 1989; Ghanem and Spanos, 1991; Kleiber and Hien, 1992; Li and Chen, 2009) includes randomness in the described factors, and for complex systems it needs to be considered by numerical methods because analytical solutions are in many cases impossible.

There are three main trends in the analysis with random variables: perturbation approach (Greene et al., 2011; Liu et al., 2013), Monte Carlo simulation (Fishman, 1995; Rubinstein 
and Kroese, 2008), and Neumann's expansion (Liu et al., 1986). The goal of this paper is a numerical nonstatistical analysis of statics and time-independent sensitivity for MDOF systems by random parameters. Starting from the stochastic version of the equilibrium equation, using the mean-point second-order perturbation method, the equations for the first two probabilistic moments of the sensitivity gradients with respect to the design parameters are derived, where the means and cross-covariances of random variables are treated as the input data. Thereby, not only the deterministic values of static response sensitivity are obtained but also the accuracy of the received results as expected values and cross-covariances. These formulations are illustrated by numerical examples.

\section{Formulation of the stochastic sensitivity for statics in the finite element context}

The objective of structural sensitivity analysis is to consider the impact of design variable changes on the system response. In this paper, the design variable vector is denoted by $\mathbf{b}=\left\{b_{a}\right\}$, $a=1,2, \ldots, A$. It can be cross-sectional areas of structural elements, thickness of a shell or plate, mass density, Young's modulus etc., while displacements, stresses or natural frequencies in main nodes can be treated as structural response measures.

The result of deterministic computation is only one variable at a given point, while the stochastic analysis determines also the accuracy of the obtained value. During the second process, the system is described by random variables. The time-independent random variable vector is assumed in the form $\mathbf{h}=\left\{h_{r}\right\}, r=1,2, \ldots, \widehat{r}$ and defined by means $\bar{h}_{r}=\mathrm{E}\left[h_{r}\right]$ and cross-covariances $\operatorname{Cov}\left(h_{r}, h_{s}\right), r, s=1,2, \ldots, \widehat{r}$. From the definition, given by Kleiber and Hien (1992), the first probabilistic moment of the random variable is expressed by

$$
\mathrm{E}\left[h_{r}\right]=\int_{-\infty}^{+\infty} h_{r} p\left(h_{r}\right) d h_{r}
$$

and the second

$$
\operatorname{Cov}\left(h_{r}, h_{s}\right)=\mathrm{E}\left[\left(h_{r}-\bar{h}_{r}\right)\left(h_{s}-\bar{h}_{s}\right)\right]=\alpha_{r} \alpha_{s} \bar{h}_{r} \bar{h}_{s} \mu\left(h_{r}, h_{s}\right)
$$

where

$$
\mu\left(h_{r}, h_{s}\right)=\int_{-\infty}^{+\infty} \int_{-\infty}^{+\infty} h_{r} h_{s} p\left(h_{r}, h_{s}\right) d h_{r} d h_{s}
$$

and

$$
\alpha_{r}=\frac{\sqrt{\operatorname{Var}\left(h_{r}\right)}}{\bar{h}_{r}}=\frac{\sigma\left(h_{r}\right)}{\bar{h}_{r}}
$$

In Equations (2.1)-(2.4) $p\left(h_{r}\right), p\left(h_{r}, h_{s}\right), \mu\left(h_{r}, h_{s}\right)$, $\operatorname{Var}\left(h_{r}\right), \sigma\left(h_{r}\right)$ and $\alpha$ denote the probability density function, joint probability density function, adopted function of correlation, variance, standard deviation and the coefficient of variation, respectively.

In this paper from this moment forth, the summation notation is included. In the following equations, two repeated indices imply the sum, which results in greater transparency of presentation. Let us consider a linear elastic complex structure with $N$ degrees of freedom. The static response of the system can be formulated then by the functional (Kleiber and Hien, 1992)

$$
\phi=G\left[q_{\alpha}\left(b_{a}, h_{r}\right), b_{a}\right] \quad \alpha=1,2, \ldots, N
$$


where $q_{\alpha}$ is determined as the time-independent vector of generalized coordinates. In the stochastic analysis of systems with multi degrees of freedom (MDOF), the stiffness matrix $K_{\alpha \beta}$ and the nodal load vector $Q_{\alpha}$ are explicit functions of both the design parameter and the random variable vector. In the static case, the considered system is time-independent, therefore damping and mass effects are omitted during computations. Consequently, the equilibrium equation is written in the stochastic form as

$$
K_{\alpha \beta}\left(b_{a}, h_{r}\right) q_{\beta}\left(b_{a}, h_{r}\right)=Q_{\alpha}\left(b_{a}, h_{r}\right) \quad \alpha, \beta=1,2, \ldots, N
$$

A solution to Eq. (2.6) with respect to $q_{\beta}$ (Eq. (2.7)) proves that the nodal displacement vector is an implicit function of $b_{a}$ and $h_{r}$

$$
q_{\beta}\left(b_{a}, h_{r}\right)=K_{\alpha \beta}^{-1}\left(b_{a}, h_{r}\right) Q_{\alpha}\left(b_{a}, h_{r}\right)
$$

To evaluate the probabilistic distribution of the static structural response with respect to the design parameters, we tend to obtain an absolute partial derivative of the functional $\phi$ with respect to the design variable, i.e. $d \phi / d b_{a}$. Assuming that the stiffness matrix and the nodal load vector are twice continuously differentiable with respect to $b_{a}$, the chain rule of differentiation gives

$$
\frac{d \phi}{d b_{a}}=\frac{\partial G}{\partial b_{a}}+\frac{\partial G}{\partial q_{\alpha}} \frac{d q_{\alpha}}{d b_{a}} \quad \alpha=1,2, \ldots, N \quad a=1,2, \ldots, A
$$

According to Eq. (2.5), $G$ is an explicit function of $b_{a}$ and $q_{\alpha}$, therefore, the partial derivatives $\partial G / \partial b_{a}$ and $\partial G / \partial q_{\alpha}$ are known. The goal of the procedure is to find the absolute partial derivative of the general coordinate vector with respect to the design variable, i.e. $d q_{\alpha} / d b_{a}$. To formulate the static sensitivity problem, the so-called adjoint system method is used (Kleiber and Hien, 1992; Choi and Kim, 2010). The adjoint equation system is adopted in the form

$$
K_{\alpha \beta} \lambda_{\beta}=\frac{\partial G}{\partial q_{\alpha}} \quad \alpha, \beta=1,2, \ldots, N
$$

Seeing that the stiffness matrix $K_{\alpha \beta}$ and the vector $\partial G / \partial q_{\alpha}$ are functions of the design and random variable, the solution to Eq. (2.9) with respect to $\lambda_{\beta}$ written as

$$
\lambda_{\beta}\left(b_{a}, h_{r}\right)=K_{\alpha \beta}^{-1}\left(b_{a}, h_{r}\right) \frac{\partial G}{\partial q_{\alpha}}\left(b_{a}, h_{r}\right)
$$

demonstrates that the adjoint variable vector is an implicit function of $b_{a}$ and $h_{r}$ and it is expressed as $\lambda=\left\{\lambda_{\alpha}\left(b_{a}, h_{r}\right)\right\}, \alpha=1,2, \ldots, N$. Differentiating Eq. (2.6) with respect to the design variable and then solving it to obtain the vector $d q_{\alpha} / d b_{a}$ and putting it into Eq. (2.8) leads to

$$
\frac{d \phi}{d b_{a}}=\frac{\partial G}{\partial b_{a}}+K_{\alpha \beta}^{-1} \frac{\partial G}{\partial q_{\alpha}}\left(\frac{\partial Q_{\alpha}}{\partial b_{a}}-\frac{\partial K_{\alpha \beta}}{\partial b_{a}} q_{\beta}\right)
$$

Substituting Eq. (2.10) into Eq. (2.11) we receive the equation for design sensitivity of the system (Kleiber and Hien, 1992)

$$
\frac{d \phi}{d b_{a}}=\frac{\partial G}{\partial b_{a}}+\lambda_{\alpha}\left(\frac{\partial Q_{\alpha}}{\partial b_{a}}-\frac{\partial K_{\alpha \beta}}{\partial b_{a}} q_{\beta}\right)
$$

It should be noted that all terms in Eq. (2.12) are simultaneously functions of the design parameter and the random variable vectors. Now, all elements from Eqs. (2.6) and (2.9) are expanded in power series around the means $\bar{h}_{r}$, in accordance with the following equation

$$
(\cdot)=(\cdot)(\overline{\mathbf{h}})+\left[\frac{\partial(\cdot)}{\partial h_{r}} \delta h_{r}+\frac{1}{2} \frac{\partial^{2}(\cdot)}{\partial h_{r} \partial h_{s}} \delta h_{r} \delta h_{s}\right]_{\mathbf{h}=\overline{\mathbf{h}}} \quad r, s=1,2, \ldots, \widehat{r}
$$


where $\delta h_{r}$ denotes the first variations $h_{r}$ about the means $\bar{h}_{r}$, and for any small parameter $\epsilon$ it can be written as (Hien, 2003)

$$
\delta h_{r}=\epsilon\left(h_{r}-\bar{h}_{r}\right)
$$

while $\delta h_{r} \delta h_{s}$ is called the second mixed variation of $h_{r}$ and $h_{s}$ about their means $\bar{h}_{r}$ and $\bar{h}_{s}$

$$
\delta h_{r} \delta h_{s}=\epsilon^{2}\left(h_{r}-\bar{h}_{r}\right)\left(h_{s}-\bar{h}_{s}\right)
$$

All the functions of the random variable are expanded in Taylor series up to the second order and substituted to Eqs. (2.6) and (2.9). After rearranging the equations and comparing terms with the same order of $\epsilon$, we receive the primary and adjoint systems of equations (compare, Kleiber and Hien, 1992):

- one pair of systems of $N$ equations of the zeroth-order

$$
K_{\alpha \beta} q_{\beta}=Q_{\alpha} \quad K_{\alpha \beta} \lambda_{\beta}=\frac{\partial G}{\partial q_{\alpha}}
$$

- $\widehat{r}$ pairs of systems of $N$ equations of the first-order

$$
K_{\alpha \beta} \frac{d q_{\beta}}{d h_{r}}=\frac{\partial Q_{\alpha}}{\partial h_{r}}-\frac{\partial K_{\alpha \beta}}{\partial h_{r}} q_{\beta} \quad K_{\alpha \beta} \frac{d \lambda_{\beta}}{d h_{r}}=\frac{\partial^{2} G}{\partial h_{r} \partial q_{\alpha}}-\frac{\partial K_{\alpha \beta}}{\partial h_{r}} \lambda_{\beta}
$$

— one pair of systems of $N$ equations of the second-order

$$
\begin{aligned}
& \left.K_{\alpha \beta} \frac{d^{2} q_{\beta}}{d h_{r} d h_{s}}\right|_{\mathbf{h}=\overline{\mathbf{h}}} \operatorname{Cov}\left(h_{r}, h_{s}\right)=\left[\frac{\partial^{2} Q_{\alpha}}{\partial h_{r} \partial h_{s}}-\frac{\partial^{2} K_{\alpha \beta}}{\partial h_{r} \partial h_{s}} q_{\beta}-2 \frac{\partial K_{\alpha \beta}}{\partial h_{r}} \frac{d q_{\beta}}{d h_{s}}\right]_{\mathbf{h}=\overline{\mathbf{h}}} \operatorname{Cov}\left(h_{r}, h_{s}\right) \\
& \left.K_{\alpha \beta} \frac{d^{2} \lambda_{\beta}}{d h_{r} d h_{s}}\right|_{\mathbf{h}=\overline{\mathbf{h}}} \operatorname{Cov}\left(h_{r}, h_{s}\right)=\left[\frac{\partial^{3} G}{\partial h_{r} \partial h_{s} \partial q_{\alpha}}-\frac{\partial^{2} K_{\alpha \beta}}{\partial h_{r} \partial h_{s}} \lambda_{\beta}-2 \frac{\partial K_{\alpha \beta}}{\partial h_{r}} \frac{d \lambda_{\beta}}{d h_{s}}\right]_{\mathbf{h}=\overline{\mathbf{h}}} \operatorname{Cov}\left(h_{r}, h_{s}\right)
\end{aligned}
$$

From the definition (Kleiber and Hien, 1992), the first probabilistic moment for the sensitivity gradient is given by

$$
\mathrm{E}\left[\frac{d \phi}{d b_{a}}\right]=\underbrace{\int_{-\infty}^{+\infty} \int_{-\infty}^{+\infty} \cdots \int_{-\infty}^{+\infty} \frac{d \phi}{d b_{a}}}_{A-\text { fold }} p_{A}\left(b_{1}, b_{2}, \ldots, b_{A}\right) d b_{1} d b_{2} \cdots d b_{A}
$$

while the second probabilistic moment is expressed analogically as

$$
\operatorname{Cov}\left(\frac{d \phi}{d b_{a}}, \frac{d \phi}{d b_{b}}\right)=\mathrm{E}\left[\left(\frac{d \phi}{d b_{a}}-\mathrm{E}\left[\frac{d \phi}{d b_{a}}\right]\right)\left(\frac{d \phi}{d b_{b}}-\mathrm{E}\left[\frac{d \phi}{d b_{b}}\right]\right)\right]
$$

The mean value of Eq. (2.12) may be written as

$$
\mathrm{E}\left[\frac{d \phi}{d b_{a}}\right]=\mathrm{E}\left[\frac{\partial G}{\partial b_{a}}\right]+\mathrm{E}\left[\lambda_{\alpha} \frac{\partial Q_{\alpha}}{\partial b_{a}}\right]-\mathrm{E}\left[\lambda_{\alpha} \frac{\partial K_{\alpha \beta}}{\partial b_{a}} q_{\beta}\right]
$$

Substituting Eq. (2.12) into Eq. (2.21) gives an expression for the second probabilistic moment of the design sensitivity gradient in the form

$$
\begin{gathered}
\operatorname{Cov}\left(\frac{d \phi}{d b_{a}}, \frac{d \phi}{d b_{b}}\right)=\mathrm{E}\left[\left(\frac{\partial G}{\partial b_{a}}+\lambda_{\alpha}\left(\frac{\partial Q_{\alpha}}{\partial b_{a}}-\frac{\partial K_{\alpha \beta}}{\partial b_{a}} q_{\beta}\right)-\mathrm{E}\left[\frac{d \phi}{d b_{a}}\right]\right)\right. \\
\left.\cdot\left(\frac{\partial G}{\partial b_{b}}+\lambda_{\gamma}\left(\frac{\partial Q_{\gamma}}{\partial b_{b}}-\frac{\partial K_{\gamma \delta}}{\partial b_{b}} q_{\delta}\right)-\mathrm{E}\left[\frac{d \phi}{d b_{b}}\right]\right)\right]
\end{gathered}
$$


All functions of the random variables from Eqs. (2.21) and (2.22) are expanded in Taylor series in accordance with Eq. (2.13) and are premultiplied. Excluding members in orders higher than the second and averaging the other terms, leads to an expression of the mean values of the design sensitivity gradient, cf. Hien and Kleiber (1991), Kleiber and Hien (1992)

$$
\begin{aligned}
\mathrm{E}\left[\frac{d \phi}{d b_{a}}\right] & =\left[\frac{\partial G}{\partial b_{a}}+\lambda_{\alpha} \mathcal{A}_{\alpha a}\right. \\
& \left.+\frac{1}{2}\left(\frac{\partial^{3} G}{\partial h_{r} \partial h_{s} \partial b_{a}}+\frac{d^{2} \lambda_{\alpha}}{d h_{r} d h_{s}} \mathcal{A}_{\alpha a}+2 \frac{d \lambda_{\alpha}}{d h_{r}} \mathcal{B}_{\alpha s a}+\lambda_{\alpha} \mathcal{C}_{\alpha r s a}\right) \operatorname{Cov}\left(h_{r}, h_{s}\right)\right]_{\mathbf{h}=\overline{\mathbf{h}}}
\end{aligned}
$$

where, for clarity purposes, the following equations are used

$$
\begin{aligned}
& \mathcal{A}_{\alpha a}=\frac{\partial Q_{\alpha}}{\partial b_{a}}-\frac{\partial K_{\alpha \beta}}{\partial b_{a}} q_{\beta} \\
& \mathcal{B}_{\alpha r a}=\frac{\partial^{2} Q_{\alpha}}{\partial h_{r} \partial b_{a}}-\frac{\partial^{2} K_{\alpha \beta}}{\partial h_{r} \partial b_{a}} q_{\beta}-\frac{\partial K_{\alpha \beta}}{\partial b_{a}} \frac{d q_{\beta}}{d h_{r}} \\
& \mathcal{C}_{\alpha r s a}=\frac{\partial^{3} Q_{\alpha}}{\partial h_{r} \partial h_{s} \partial b_{a}}-\frac{\partial^{3} K_{\alpha \beta}}{\partial h_{r} \partial h_{s} \partial b_{a}} q_{\beta}-2 \frac{\partial^{2} K_{\alpha \beta}}{\partial h_{r} \partial b_{a}} \frac{d q_{\beta}}{d h_{s}}-\frac{\partial K_{\alpha \beta}}{\partial b_{a}} \frac{d^{2} q_{\beta}}{d h_{r} d h_{s}}
\end{aligned}
$$

The cross-covariances at $d \phi / d b_{a}$ and $d \phi / d b_{b}$ are

$$
\begin{aligned}
\operatorname{Cov} & \left(\frac{d \phi}{d b_{a}}, \frac{d \phi}{d b_{b}}\right)=\left[\left(\frac{\partial^{2} G}{\partial h_{r} \partial b_{a}} \frac{\partial^{2} G}{\partial h_{s} \partial b_{b}}+\left(\mathcal{A}_{\beta b} \mathcal{B}_{\alpha a s}+\mathcal{A}_{\alpha a} \mathcal{B}_{\beta b s}\right) \lambda_{\alpha} \frac{d \lambda_{\beta}}{d h_{r}}\right.\right. \\
& -\left(\frac{\partial G}{\partial b_{a}} \mathcal{C}_{\beta r s b}+\frac{\partial^{2} G}{\partial h_{r} \partial b_{a}} \mathcal{B}_{\beta r b}+\frac{\partial^{3} G}{\partial h_{r} \partial h_{s} \partial b_{a}} \mathcal{A}_{\beta b}\right) \lambda_{\beta} \\
& -\left(\frac{\partial G}{\partial b_{b}} \mathcal{C}_{\alpha r s a}+\frac{\partial^{2} G}{\partial h_{r} \partial b_{b}} \mathcal{B}_{\alpha r a}+\frac{\partial^{3} G}{\partial h_{r} \partial h_{s} \partial b_{b}} \mathcal{A}_{\alpha a}\right) \lambda_{\alpha} \\
& -\left(2 \frac{\partial G}{\partial b_{a}} \mathcal{B}_{\alpha s b}+\frac{\partial^{2} G}{\partial h_{s} \partial b_{a}} \mathcal{A}_{\alpha b}+2 \frac{\partial G}{\partial b_{b}} \mathcal{B}_{\alpha r a}+\frac{\partial^{2} G}{\partial h_{s} \partial b_{b}} \mathcal{A}_{\alpha a}\right) \frac{\lambda_{\alpha}}{d h_{r}} \\
& \left.-\left(\frac{\partial G}{\partial b_{b}} \mathcal{A}_{\alpha a}+\frac{\partial G}{\partial b_{a}} \mathcal{A}_{\alpha b}\right) \frac{d^{2} \lambda_{\alpha}}{d h_{r} d h_{s}}+\lambda_{\alpha} \lambda_{\beta} \mathcal{B}_{\alpha a r} \mathcal{B}_{\beta b s}+\mathcal{A}_{\alpha a} \mathcal{A}_{\beta b} \frac{d \lambda_{\alpha}}{d h_{r}} \frac{d \lambda_{\beta}}{d h_{s}}\right) \operatorname{Cov}\left(h_{r}, h_{s}\right) \\
& -\frac{1}{4}\left(\frac{\partial^{3} G}{\partial h_{t} \partial h_{u} \partial b_{b}}+2 \frac{d \lambda_{\beta}}{d h_{t}} \mathcal{B}_{\beta u b}+\mathcal{A}_{\beta b} \frac{d^{2} \lambda_{\beta}}{d h_{t} d h_{u}}+\lambda_{\beta} \mathcal{C}_{\beta t u b}\right) \\
& \left.\cdot\left(\frac{\partial^{3} G}{\partial h_{r} \partial h_{s} \partial b_{a}}+\frac{d^{2} \lambda_{\alpha}}{d h_{r} d h_{s}} \mathcal{A}_{\alpha a}+2 \frac{d \lambda_{\alpha}}{d h_{r}} \mathcal{B}_{\alpha s a}+\lambda_{\alpha} \mathcal{C}_{\alpha r s a}\right) \operatorname{Cov}\left(h_{r}, h_{s}\right) \operatorname{Cov}\left(h_{t}, h_{u}\right)\right]_{\mathbf{h}=\overline{\mathbf{h}}}
\end{aligned}
$$

with $r, s, t, u=1,2, \ldots, \widehat{r}$. Interestingly, Eq. (2.25) is obtained by including terms up to the second order, not only to the first like in Hien and Kleiber (1991), Kleiber and Hien (1992). The procedure of obtaining and averaging particular members of the first two probabilistic moments of the static design sensitivity can be found in detail in Weber (2014), while this paper is confined to present only the final version of these equations.

The equation for covariances at $d \phi / d b_{a}$ and $d \phi / d b_{b}$ in Weber (2014) is obtained by using the expressions for $E\left[d \phi / d b_{a}\right]$ and $E\left[d \phi / d b_{b}\right]$ as the final products. To derive Eq. (2.25), the members $E\left[d \phi / d b_{a}\right]$ and $E\left[d \phi / d b_{b}\right]$ are determined by Eq. (2.23) and substituted into Eq. (2.22). After ordering particular members, a more concise expression for the second probabilistic moment of the static design sensitivity than that presented by Weber (2014) is received.

\section{Numerical examples - results and discussion}

Deterministic and stochastic computations are executed by the finite element code POLSAP (see, Hien and Kleiber, 1990), properly adapted for this type of analysis. At the beginning of 
numerical illustrations, let us consider a three-bar truss system. The analytical equations of nodal displacements and their sensitivities with respect to cross-sectional areas of the elements, were derived for the model by Choi and Kim (2010). The goal of this example is to obtain not only the deterministic displacements of nodes, but also their expected values and standard deviations by POLSAP and compare them with the results given by equations formulated by Choi and Kim (2010).
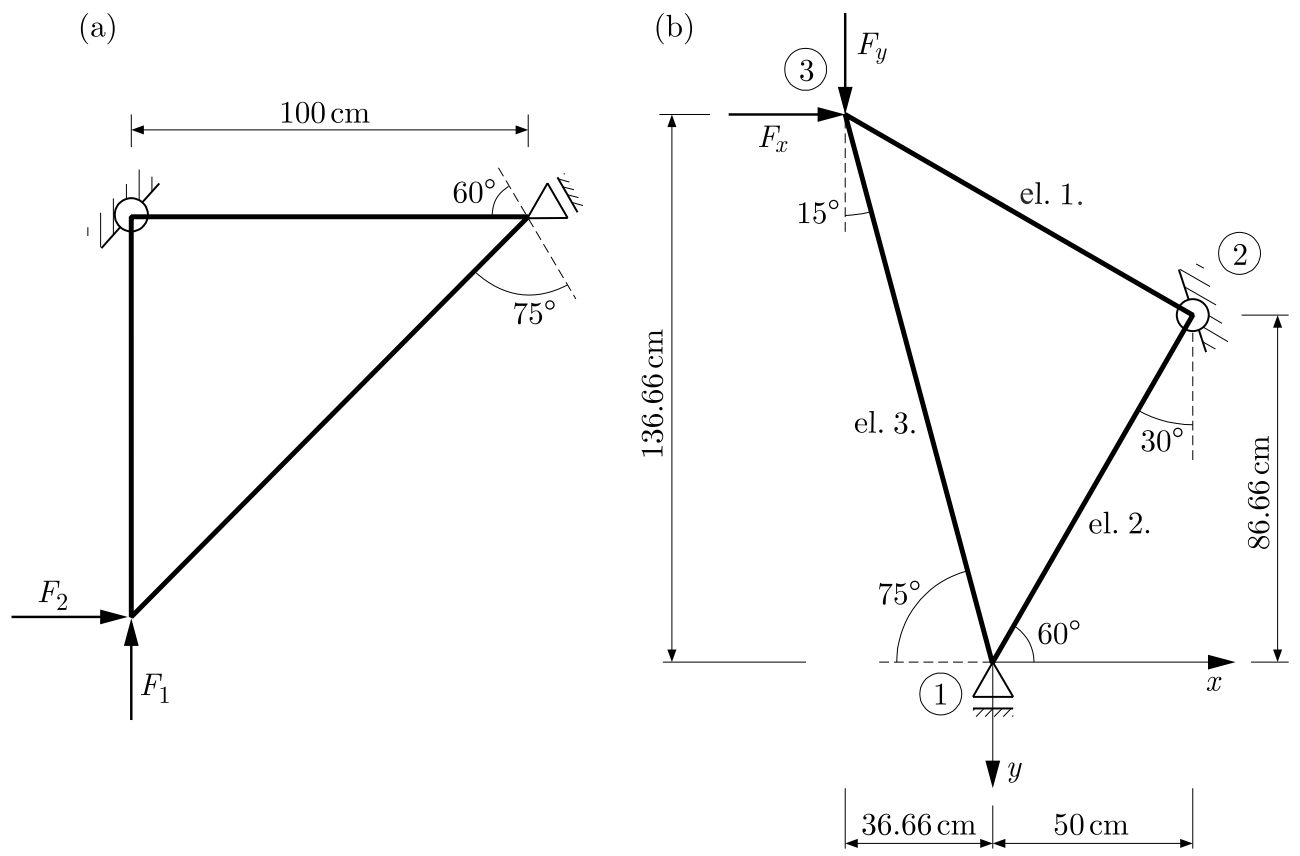

Fig. 1. Three-bar truss system (a) presented in Choi and Kim (2010), (b) adopted in numerical computation

To simplify the numerical model, the Choi and Kim system (2010) (see Fig. 1a) is rotated as shown in Fig. 1b, and nodal loads are reduced to vertical and horizontal forces $F_{x}=18.33 \mathrm{kN}$ and $F_{y}=68.30 \mathrm{kN}$ that correspond to forces $F_{1}=F_{2}=50 \mathrm{kN}$. All results shown in Tables 1 and 2 are given for the adopted global coordinate system $x y$ presented in Fig. 1b. Therefore, to compare the displacements and sensitivities, the values received by equations from literature have to be transformed by using trigonometric functions with an angle of 30 degrees.

The input data of the material and elements are adopted for simplification as: Young's modulus $E=200 \mathrm{GPa}$ and cross-sectional areas of specific bars $A_{1}=A_{2}=A_{3}=5 \mathrm{~cm}^{2}$. One bar is assumed as one truss element in the numerical model. The cross-sectional areas of particular elements are adopted as random design variables with the means $E\left[A_{r}\right]=5 \mathrm{~cm}^{2}$, and correlation function defined by the equation, cf. Kleiber and Hien (1992)

$$
\mu\left(A_{r}, A_{s}\right)=\exp \left(\frac{-\left|x_{r}-x_{s}\right|}{\lambda}\right) \exp \left(\frac{-\left|y_{r}-y_{s}\right|}{\lambda}\right)
$$

where $x_{r}, x_{s}$ and $y_{r}, y_{s}$ designates the $x$ - and $y$-coordinates of the mid-points of the next two elements in the model, respectively. The symbol $\lambda$ denotes the decay factor depending on the unit system used in numerical analysis. Its value is selected in order to receive no diagonal and non-zero covariance matrix of the random variables. For this example, $\lambda=300$ and the coefficient of variation $\alpha=0.1$ are adopted as the input data. In the second moment perturbation method, the random variables must fulfill the condition about small fluctuation and continuity at $\bar{h}_{r}$, therefore, the selection of $\alpha$-coefficient is a very important part of numerical computations, cf. Weber (2014). 
The correlation function shows a dependence between the random variables. The bigger the distance from one element to another, the smaller impact between them is observed. For the members situated at a considerable distance from each other, the described dependence tends to zero. Using Eq. (3.1), we obtain results equal to 1 on the main diagonal, and the values in the range from 0 to 1 for other elements. Additionally, the bigger the difference between the coordinates, the smaller results of the coefficient in the matrix, which satisfies the assumptions for the correlation function.

The deterministic and expected values of displacements obtained by POLSAP are similar to those given by Choi and Kim (2010) (see Table 1). The maximum difference between analytical and deterministic results is about $0.3 \%$, while that between deterministic and stochastic is $0.85 \%$.

Table 1. Non-zero nodal static displacements for the three-bar truss system, $[\mathrm{cm}]$

\begin{tabular}{|c|c|c|c|c|}
\hline \multirow[b]{2}{*}{$\begin{array}{l}\text { Node } \\
\text { number }\end{array}$} & \multirow[b]{2}{*}{ Coordinate } & \multirow[b]{2}{*}{$\begin{array}{c}\text { Analytical } \\
\text { values }\end{array}$} & \multicolumn{2}{|c|}{ POLSAP } \\
\hline & & & $\begin{array}{l}\text { Deterministic } \\
\text { displacements }\end{array}$ & $\begin{array}{c}\text { Expected } \\
\text { values }\end{array}$ \\
\hline 1 & $x$ & 0.073205 & 0.073416 & 0.073905 \\
\hline 3 & $x$ & -0.084108 & -0.084146 & -0.084863 \\
\hline 3 & $y$ & 0.145680 & 0.145868 & 0.147066 \\
\hline
\end{tabular}

The functional of the structural response defined by Eq. (2.5) is accepted in numerical analysis in the form

$$
\phi=\frac{\left|q_{\alpha}\right|}{q_{\text {all }}}-1<0
$$

where $q_{\alpha}, q_{a l l}$ are the actual and allowable displacements in a selected node. To compare the sensitivity results obtained from Choi and Kim (2010) and POLSAP, $q_{a l l}=1.0 \mathrm{~cm}$ is assumed for every node, otherwise the values given by the equations from literature should be divided by the accepted $q_{\text {all }}$.

In Table 2, displacement design sensitivities for selected nodes are presented. The maximum difference between analytical and deterministic values is $0.43 \%$. The deterministic and stochastic results of static sensitivity vary in the range of $2.5-3.0 \%$. The standard deviation is $19.4 \%$ of expected values.

Table 2. Displacement design sensitivity - bar cross-sectional areas as random design variables, $\left[1 / \mathrm{cm}^{2}\right]$

\begin{tabular}{|c|c|c|c|c|c|}
\hline \multirow{2}{*}{$\begin{array}{c}\text { Node } \\
\text { number }\end{array}$} & Displa- & Obtained values & \multicolumn{3}{|c|}{ POLSAP } \\
\cline { 3 - 6 } & direction & based on Choi & Deterministic & Expected & Standard \\
and Kim (2010) & values & values & deviations \\
\hline \hline \multicolumn{6}{|c|}{ cross-sectional area of el. no. 2 as design variable } \\
\hline \hline 1 & $\mathrm{x}$ & $-1.46410 \mathrm{E}-2$ & $-1.46831 \mathrm{E}-2$ & $-1.50737 \mathrm{E}-2$ & $2.92693 \mathrm{E}-3$ \\
3 & $\mathrm{y}$ & $-4.64102 \mathrm{E}-3$ & $-4.66079 \mathrm{E}-3$ & $-4.77790 \mathrm{E}-3$ & $9.27750 \mathrm{E}-4$ \\
\hline \multicolumn{7}{|c|}{ cross-sectional area of el. no. 3 as design variable } \\
\hline 3 & $\mathrm{x}$ & $-1.41421 \mathrm{E}-2$ & $-1.41423 \mathrm{E}-2$ & $-1.45617 \mathrm{E}-2$ & $2.82752 \mathrm{E}-3$ \\
3 & $\mathrm{y}$ & $-2.44949 \mathrm{E}-2$ & $-2.45133 \mathrm{E}-2$ & $-2.52209 \mathrm{E}-2$ & $4.89726 \mathrm{E}-3$ \\
\hline
\end{tabular}

The second numerical example is a spatial dome presented in Fig. 2 (compare Weber and Hien, 2010; Weber, 2014). The geometrical dimensions included in the analysis are: base diameter $1000 \mathrm{~cm}$ and height $500 \mathrm{~cm}$. Each bar in the structure is modelled as a one beam element with the following characteristics: Young's modulus $E=200 \mathrm{GPa}$, mass density $7.85 \mathrm{KNs}^{2} / \mathrm{m}^{4}$, Poisson's ratio $\nu=0.3$ and cross-section area $A=20 \mathrm{~cm}^{2}$. Nodes $1,3,5,7$ and 9 are supported by pins. 
For the static and sensitivity analysis, only one vertical force is taken into account $F_{z}=1000 \mathrm{kN}$. For clarity purposes, the dead weight of the structure is omitted during computations. As it is well known, this type of bar structures loaded only in nodes is usually modelled as a truss system. However, the goal of this presentation is to show the influence of complexity of the finite element mesh on static sensitivity results. Therefore, in this system, the rigid connections between bars are assumed. A comparison of the results obtained for the truss and beam model of this structure can be found in Weber (2014).

(a)

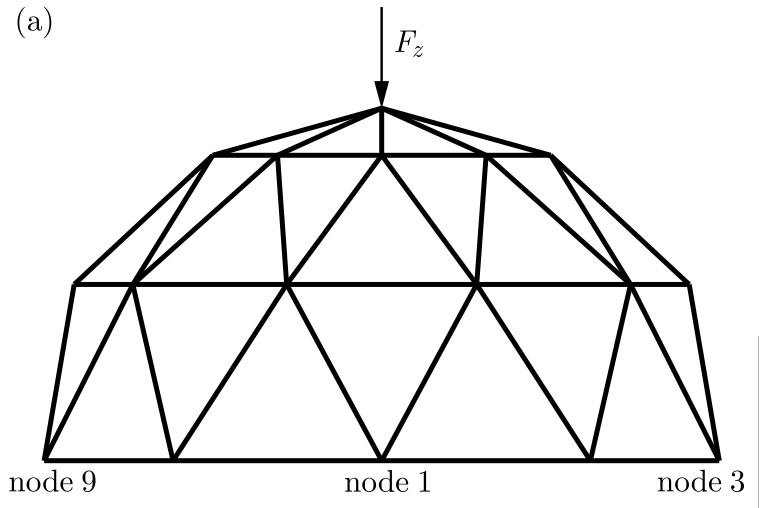

(b)

node 9

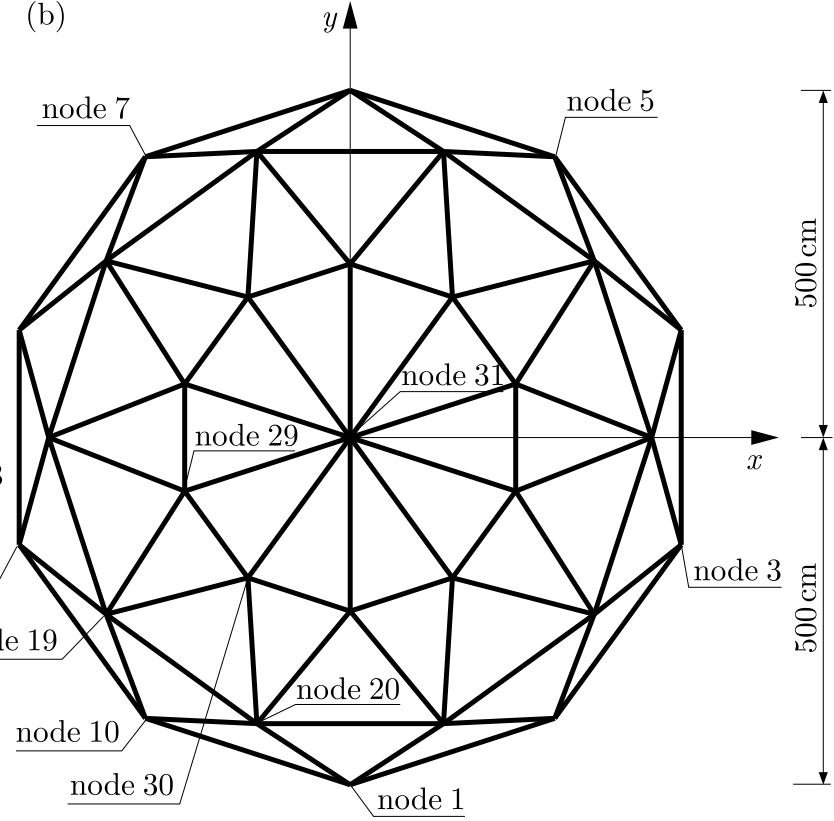

Fig. 2. Spatial dome (a) front, (b) bird eye - view

In static and sensitivity computations, the cross-sectional areas of elements are adopted as random design variables with the mean values $\bar{A}=20 \mathrm{~cm}^{2}$. The correlation function and functional response of the system are assumed as in Eqs. (3.1) and (3.2). The decay factor $\lambda=200$ and the coefficient of variation $\alpha=0.1$ are used in numerical computations.

The largest vertical deflection occurs at the top of the dome, which is predictable because of the point of force application. The deterministic and expected values of the nodal displacements in different directions vary by about $1 \%$, which is acceptable.

The considered system is symmetric in terms of geometry, supported conditions and external load. Numerical computations give the same results in corresponding nodes, which confirms that the model input to the program is correct. However, for clarity purposes, Table 3 presents the values of displacements only for selected nodes.

Table 3. Selected nodal static displacements for the spatial dome, [cm]

\begin{tabular}{|c|c|c|c|c|}
\hline $\begin{array}{c}\text { Node } \\
\text { number }\end{array}$ & Coordinate & $\begin{array}{c}\text { Deterministic } \\
\text { displacements }\end{array}$ & $\begin{array}{c}\text { Expected } \\
\text { values }\end{array}$ & $\begin{array}{c}\text { Difference } \\
{[\%]}\end{array}$ \\
\hline 31 & $z$ & -2.049777 & -2.070121 & 0.99 \\
\hline 29 & $x$ & -0.260314 & -0.262972 & 1.02 \\
\hline 30 & $x$ & -0.162236 & -0.163888 & 1.02 \\
\hline 20 & $y$ & -0.144629 & -0.145915 & 0.89 \\
\hline 19 & $y$ & -0.100229 & -0.101091 & 0.86 \\
\hline
\end{tabular}

Both deterministic and stochastic analysis of static sensitivity study the vulnerability of various node displacements with respect to cross-sectional areas of different elements. The value 
of $q_{\text {all }}$ is chosen separately for every node and movement direction, according to Eq. (3.2). The most significant results of static displacement sensitivity for selected nodes, with the crosssectional areas of elements as the random design variables, are given in Table 4 . The differences between deterministic and expected values are about 2.5-3\%, while the standard deviations are equal to $20 \%$ of expected values. In both examples, all computations have been conducted in centimeters and kilonewtons as basic units.

Table 4. Displacement design sensitivity - bar cross-sectional areas as random design variables, $\left[1 / \mathrm{cm}^{2}\right]$

\begin{tabular}{|c|c|c|c|c|c|c|}
\hline $\begin{array}{c}\text { Node } \\
\text { number }\end{array}$ & $\begin{array}{c}\text { Displ. } \\
\text { direct. }\end{array}$ & $\begin{array}{c}q_{\text {all }} \\
\text { [cm] }\end{array}$ & $\begin{array}{c}\text { Design } \\
\text { el. number }\end{array}$ & $\begin{array}{c}\text { Deterministic } \\
\text { values }\end{array}$ & $\begin{array}{c}\text { Expected } \\
\text { values }\end{array}$ & $\begin{array}{c}\text { Standard } \\
\text { deviations }\end{array}$ \\
\hline \hline 31 & $z$ & 2.5 & 72 & $-1.94449 \mathrm{E}-3$ & $-2.00421 \mathrm{E}-3$ & $3.88446 \mathrm{E}-4$ \\
10 & $z$ & 1.0 & 29 & $-2.18042 \mathrm{E}-3$ & $-2.23223 \mathrm{E}-3$ & $3.78821 \mathrm{E}-4$ \\
\hline 29 & $x$ & 1.0 & 71 & $9.89193 \mathrm{E}-3$ & $1.01891 \mathrm{E}-2$ & $1.97174 \mathrm{E}-3$ \\
30 & $x$ & 1.0 & 72 & $6.14086 \mathrm{E}-3$ & $6.32574 \mathrm{E}-3$ & $1.22490 \mathrm{E}-3$ \\
\hline 20 & $y$ & 1.0 & 32 & $-3.17786 \mathrm{E}-3$ & $-3.25960 \mathrm{E}-3$ & $5.7216 \mathrm{E}-4$ \\
19 & $y$ & 1.0 & 40 & $-3.59847 \mathrm{E}-3$ & $-3.69325 \mathrm{E}-3$ & $6.59437 \mathrm{E}-4$ \\
\hline
\end{tabular}

Considering various examples of the static displacement sensitivity allows one to spot a pattern. In most instances, a point displacement is the most sensitive with respect to, e.g., the cross-sectional areas of elements in the immediate vicinity of the considered point. However, in some cases there are certain derogations of the pattern when a displacement of a node turns out to be the most sensitive with respect to the cross-section of an element not lying directly by the examined node. Then sensitivity analysis allows one to find the key element of the considered displacement. This computation changes our view of the importance of individual structural members in the system and thereby should have a significant role in modern design.

To show the influence of finite element mesh complexity on the results of stochastic static sensitivity, the second model of the spatial dome is created, where one bar is divided into four beam elements, cf. Fig. 3. It yields a system consisting of 320 members. Thereby, we can determine not only which element but also which part of it generates the displacement at a specific point the most sensitive with respect to the cross-section area. This may be useful while examining the model in terms of the predicted path of failure or structure strengthening.

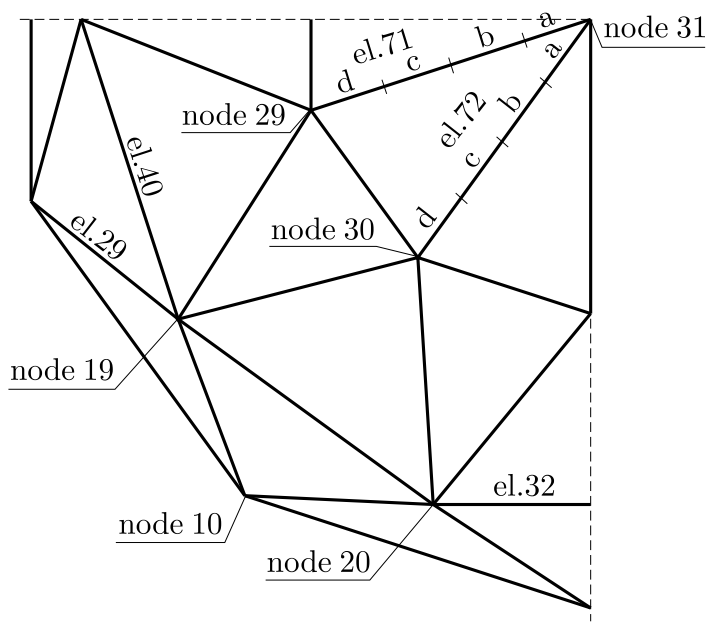

Fig. 3. Selected element number

The comparison of results received for the first and second numerical models are summarized in Table 5. It is clear that the sensitivity values obtained for the 320 element scheme is about 
four times less than for the 80 element one. It seems to be natural, because we examine the sensitivity with respect to the cross-sectional areas of the elements four times shorter than at the beginning. Careful analysis presented by Weber (2014) gives the following dependence: the higher the number of finite elements in the mesh, the smaller impact of changes in cross-section areas of elements on the nodal displacements.

Table 5. Displacement design sensitivity - bar cross-sectional areas as random design variables, $\left[1 / \mathrm{cm}^{2}\right]$

\begin{tabular}{|c|c|c|c|c|c|c|}
\hline $\begin{array}{c}\text { Node } \\
\text { number }\end{array}$ & $\begin{array}{c}\text { Displ. } \\
\text { direct. }\end{array}$ & $\begin{array}{c}q_{\text {all }} \\
\text { [cm }\end{array}$ & $\begin{array}{c}\text { Design } \\
\text { el. number }\end{array}$ & $\begin{array}{c}\text { Deterministic } \\
\text { values }\end{array}$ & $\begin{array}{c}\text { Expected } \\
\text { values }\end{array}$ & $\begin{array}{c}\text { Standard } \\
\text { deviations }\end{array}$ \\
\hline \hline 31 & $z$ & 2.5 & $72 a$ & $-5.18116 \mathrm{E}-4$ & $-5.34095 \mathrm{E}-4$ & $1.04516 \mathrm{E}-4$ \\
& & & $72 b$ & $-4.71989 \mathrm{E}-4$ & $-4.86661 \mathrm{E}-4$ & $9.51452 \mathrm{E}-5$ \\
& & & $72 c$ & $-4.62112 \mathrm{E}-4$ & $-4.76374 \mathrm{E}-4$ & $9.25389 \mathrm{E}-5$ \\
& & & $72 d$ & $-4.88431 \mathrm{E}-4$ & $-5.03158 \mathrm{E}-4$ & $9.66361 \mathrm{E}-5$ \\
\hline 29 & $x$ & 1.0 & $71 a$ & $2.41918 \mathrm{E}-3$ & $2.49298 \mathrm{E}-3$ & $4.87353 \mathrm{E}-4$ \\
& & & $71 b$ & $2.50572 \mathrm{E}-3$ & $2.58152 \mathrm{E}-3$ & $5.02619 \mathrm{E}-4$ \\
& & & $71 c$ & $2.51620 \mathrm{E}-3$ & $2.59250 \mathrm{E}-3$ & $5.05291 \mathrm{E}-4$ \\
& & & $71 d$ & $2.45059 \mathrm{E}-3$ & $2.52538 \mathrm{E}-3$ & $4.94002 \mathrm{E}-4$ \\
\hline
\end{tabular}

\section{Concluding remarks}

In the stochastic static sensitivity analysis by using the second order perturbation method, the same member, for example the cross-section area of an element, is both the design and random variable. It is significant in terms of the cost of numerical computations. It allows us to obtain complex results in the form of deterministic and expected values, and standard deviations. On the basis of the presented formulation, using the first two perturbation moments of random variables as the input data, we obtain the means and cross-covariances of the static design sensitivity with the second order accuracy. We receive results with the same precision as those in the Monte Carlo simulation, but by considering an $\widehat{r}$ order system of equations no $\widehat{r}^{3}$.

It is known that even small uncertainties in design parameters may have a large influence on results of displacements and internal forces, therefore this type of analysis seems to be very important in the design. Sensitivity analysis sometimes can provide a completely new insight into the work of a structure and the meaning of particular members in the considered system. It allows one to find the most sensitive point that determines the stability of the entire system.

In this paper, only design sensitivity with random parameters for the static case is presented. It seems that dynamic computations need to be an integral part of analysis of complex structures. Only by using both static and dynamic stochastic design sensitivity, we can determine the optimal solution for a system while taking into account all relevant aspects. Therefore, the dynamic sensitivity by deterministic and random parameters will be the subject of further work.

\section{Acknowledgments}

I would like to express my gratitude to promoter of my doctoral dissertation Professor Tran Duong Hien, for providing a computer programme for this research.

\section{References}

1. Adomian G., 1983, Stochastic Systems, Academic Press

2. Bathe K.-J., 1982, Finite Element Procedures in Engineering Analysis, Prentice-Hall 
3. Choi K.K., Kim N.-H., 2010, Structural Sensitivity Analysis and Optimization, Springer

4. Ding J., PAn Z., Chen L., 2012, Parameter identification of multibody systems based on second order sensitivity analysis, International Journal of Non-Linear Mechanics, 47, 1105-1110

5. Drewko J., Hien T.D., 2005, First- and second-order sensitivities of beams with respect to cross-sectional cracks, Archive of Applied Mechanics, 74, 309-324

6. Fishman G.S., 1995, Monte Carlo: Concept, Algoritms and Aplications, Springer

7. Ghanem R.G., Spanos P.D., 1991, Stochastic Finite Elements: A Spectral Approach, Springer

8. Greene M.S., Liu Y., Chen W., LiU W.K., 2011, Computational uncertainty analysis in multiresolution materials via stochastic constitutive theory, Computer Methods in Applied Mechanics and Engineering, 200, 309-325

9. Haug E.J., Choi K.K., Komkov V., 1986, Design Sensitivity Analysis of Structural Systems, Academic Press

10. Hien T.D., 2003, Numerical Analysis of Stochastic Systems, Wyd. PS

11. Hien T.D., Kleiber M., 1989, Computational aspects in structural design sensitivity analysis for statics and dynamics, Computers and Structures, 33, 939-950

12. Hien T.D., Kleiber M., 1990, POLSAP - A Finite Element Code for Deterministic and Stochastic Analyses of Large 3D Structures, IPPT PAN

13. Hien T.D., Kleiber M., 1991, Stochastic structural design sensitivity of static response, Computers and Structures, 38, 5/6, 659-667

14. Hisada T., NAKagiri S., 1981, Stochastic finite element method for structural safety and relability, Proceedings of 3rd International Conference on Structural Safety and Reliability, 395-402

15. Kincayd D., Cheney W., 2002, Numerical Analysis. Mathematic of Scientific Computing, 3rd ed., Wadsworth Group

16. Kleiber M., Hien T.D., 1992, The Stochastic Finite Element Method, Willey

17. Leet K.M., Uang Ch.-M., GilbertA.M., 2010, Fundamentals of Structural Analysis, 4th ed., Mc Graw-Hill

18. Li J., Chen J., 2009, Stochastic Dynamics of Structures, Wiley

19. Liu W.K., Belytschko T., Mani A., 1986, Random field finite elements, International Journal for Numerical Methods in Engineering, 23, 1831-1845

20. Liu Y., Greene M.S., Chen W., Dikin A.D., Liu W.K., 2013, Computational microstructure characterization and reconstruction for stochastic multiscale material design, Computer-Aided Design, 65-76

21. Mroz Z., Bojczuk D., 2012, Shape and topology sensitivity analysis and its application to structural design, Archive of Applied Mechanics, 82, 1541-1555

22. Mroz Z., Haftka R.T., 1986, First- and second-order sensitivity analysis of linear and nonlinear systems, AIAA Journal, 24, 1187-1192

23. NiczyJ J., 2003, Multi-Criteria Optimization of Reliability and Estimation of the Technical State of Bar Structures Using Fuzzy Sets Theory (in Polish), Wyd. PS

24. Rubinstein R.Y., Kroese D. P., 2008, Simulation and the Monte Carlo Method, 2nd ed., Willey

25. Spanos P.D., Ghanem R.G., 1989, Stochastic finite element expansion for random media, Journal of Engineering Mechanics, 115, 5, 1035-1053

26. Weber H., Hien T.D., 2010, Elimination of beat effects in structures by added lumped mass, Pomiary Automatyka Kontrola, 56, 617-619 
27. Weber H., 2014, Numerical analysis of static and dynamic sensitivity of complex structural systems with random parameters, Ph.D Thesis, West Pomeranian University of Technology Szczecin

28. Zienkiewicz O.C., Taylor R.M., 1991, The Finite Element Method, McGraw-Hill

Manuscript received May 18, 2015; accepted for print November 24, 2015 